\title{
Validation of downregulated microRNAs during osteoclast formation and osteoporosis progression
}

\author{
YAN MA ${ }^{1,2}$, ZHI SHAN ${ }^{1,2}$, JIANJUN MA $^{1,2}$, QIANG WANG ${ }^{1,2}$, JUNJIE CHU $^{1,2}$, \\ PEIWEI XU ${ }^{1,2}$, AN QIN ${ }^{3}$ and SHUNWU FAN ${ }^{1,2}$ \\ ${ }^{1}$ Department of Orthopaedics, Sir Run Run Shaw Hospital, School of Medicine, Zhejiang University; \\ ${ }^{2}$ Department of Orthopaedics, Sir Run Run Shaw Institute of Clinical Medicine of Zhejiang University, Hangzhou, \\ Zhejiang 310016; ${ }^{3}$ Department of Orthopaedics, Shanghai Key Laboratory of Orthopaedic Implants, \\ Shanghai Ninth People's Hospital, Shanghai Jiaotong University School of Medicine, \\ Shanghai 200011, P.R. China
}

Received February 4, 2015; Accepted December 14, 2015

DOI: $10.3892 / \mathrm{mmr} .2016 .4765$

\begin{abstract}
Enhanced osteoclast formation and function have essential roles during post-menopausal osteoporosis. A number of cytokines have been reported to regulate osteoclastogenesis and to be involved during the pathogenesis of osteoporosis. However, the regulation of osteolysis by microRNAs (miRNAs) has remained to be fully elucidated. The present study used a microarray analysis to identify a variety of miRNAs that are differentially expressed during osteoclast formation. Six down-regulated miRNAs, miR-21a-5p, miR-27a-3p, let-7i-5p, miR-22-3p, miR-340-5p and miR-23a-5p, whose molecular mechanisms during osteoclast differentiation have not been reported previously, were further assessed. Using an osteoclast formation assay and a mouse model of progressive osteoporosis, the downregulation of these miRNAs was validated in vitro and in vivo. Of note, the expression patterns of these six miRNAs were associated with the progression of osteoporosis. Therefore, these miRNAs are of potential diagnostic and therapeutic value for osteolytic diseases.
\end{abstract}

Correspondence to: Professor Shunwu Fan, Department of Orthopaedics, Sir Run Run Shaw Hospital, School of Medicine, Zhejiang University, 3 Qingchun Road East, Hangzhou, Zhejiang 310016, P.R. China

E-mail: orthopaedics2009@hotmail.com

Dr An Qin, Department of Orthopaedics, Shanghai Key Laboratory of Orthopaedic Implants, Shanghai Ninth People's Hospital, Shanghai Jiaotong University School of Medicine, 639 Zhizaoju Road, Shanghai 200011, P.R. China

E-mail: dr_qinan@163.com

Key words: microRNA, downregulated, osteoclast, osteoporosis, bone

\section{Introduction}

Osteoporosis occurs due to an imbalanced homeostasis between bone resorption osteoclasts and bone formation osteoblasts and represents a global health problem. The low bone mineral density observed in post-menopausal women is mainly due to enhanced osteoclast formation and/or function. Previous studies have demonstrated that numerous key cytokines have important roles in osteoclastogenesis and osteoporosis, including macrophage colony-stimulating factor (M-CSF), receptor activator of nuclear factor- $\kappa \mathrm{B}$ ligand (RANKL), interleukin 1 and tumor necrosis factor $\alpha$ (1-3). Consequently, several of these key cytokines have been developed as therapeutic targets for the treatment of osteoporosis. For instance, denosumab, which targets RANKL, has been proved to effectively prevent osteoporosis in post-menopausal women (4).

Apart from cytokines, microRNAs (miRNAs/miRs) have essential roles in regulating cell biology and have been proposed as potential biomarkers or therapeutic targets for several diseases (5-7). miRNAs are non-coding small RNAs containing 22 nucleotides. By suppressing translation of the targeted mRNAs, they are estimated to control more than one third of all human genes (8). Previous studies have demonstrated that miRNAs have critical roles in regulating osteoclastogenesis and are associated with numerous osteolytic diseases (9-14). Considering the possible adverse effects of the synthetic drugs currently used for the treatment of these diseases, miRNAs have received increased attention as possible alternative therapeutic targets for the prevention and treatment of osteolytic diseases such as osteoporosis $(13,14)$. Therefore, the present study aimed to assess differentially expressed miRNAs during osteoclastogenesis in vitro and in ovariectomy (OVX)-induced osteoporosis in vivo. More specifically, the present study focused on the downregulated miRNAs, which were associated with the progression of osteoporosis in vivo.

\section{Materials and methods}

Mediaandreagents. The $\alpha$-modifiedEagle's medium ( $\alpha$-MEM), foetal bovine serum (FBS) and penicillin/streptomycin were 
obtained from Gibco (Thermo Fisher Scientific, Inc., Waltham, MA, USA). The Cell Counting kit-8 (CCK-8) was purchased from Dojindo Molecular Technology (Kumamoto, Japan). Mouse RANKL and recombinant soluble mouse M-CSF were obtained from R\&D Systems (Minneapolis, MN, USA). The tartrate-resistant acid phosphatase (TRAP) staining kit, TRIzol, chloral hydrate and ketamine were purchased from Sigma-Aldrich (St Louis, MO, USA). All other reagents were from Sigma-Aldrich, unless stated otherwise. The present study was approved by the ethics committee of Sir Run Run Shaw Hospital (Hangzhou, China).

Bone marrow cell culture. Bone marrow macrophages (BMMs) were separated from the femurs and tibiae of two six-week-old female C57BL/6 mice (Shanghai SLAC Laboratory Animal Co., Ltd., Shanghai, China) as described previously (15). The isolated BMMs were then cultured in $\alpha$-MEM containing $10 \% \mathrm{FBS}$ and $30 \mathrm{ng} / \mathrm{ml} \mathrm{M}-\mathrm{CSF}$ for proliferation. Cells were harvested when reaching $90 \%$ confluence and seeded into 96-well plates at a density of $8 \times 10^{3}$ cells/well or into six-well plates at a density of $8 \times 10^{5}$ cells/well in triplicate. After incubation for $24 \mathrm{~h}$, cells were treated with RANKL $(50 \mathrm{ng} / \mathrm{ml})$ for various durations $(0,1,3,5$ or 7 days). Cells in 96 -well plates were then fixed and stained for TRAP activity following the kit manufacturer's instructions. Cells in the six-well plates were dissolved in TRIzol for extraction of total RNA (including miRNA).

Animal model. An OVX-induced osteoporosis model was used to establish various degrees of osteoporosis in vivo. All experimental procedures were approved by the Animal Care and Experiment Committee of Zhejiang University School of Medicine (Huangzhou, China), and the study was performed according to the guidelines for Ethical Conduct from the Care and Use of Nonhuman Animals in Research by the American Psychological Association. Healthy eight-week-old C57BL/6J mice $(n=24)$ were subjected to sham operation $(n=8)$ or ovariectomy (OVX) $(\mathrm{n}=16)$ under anesthesia using $8 \%$ chloral hydrate. The mice were housed in three cages in an animal facility with conditions of $22-24^{\circ} \mathrm{C}, 12$-h light/dark cycle, 50-60\% humidity with access to food and water ad libitum. Eight sham-operated mice and eight OVX mice were sacrificed by euthanasia with $0.8 \mathrm{ml} / 100 \mathrm{~g}$ body weight ketamine delivered by intraperitoneal injection at 1 month following surgery (sham and OVX 1). The remaining eight OVX mice were sacrificed as above at 2 months following surgery (OVX 2). The left tibiae were obtained and fixed in $4 \%$ paraformaldehyde for micro-computer tomography $(\mathrm{CT})$ analysis, while the right tibiae were immediately snap-frozen in liquid nitrogen for RNA (miRNA and mRNA) isolation.

Micro-CT analysis. After being fixed with 4\% paraformaldehyde for $48 \mathrm{~h}$, the left tibiae of each mouse were washed with phosphate-buffered saline (PBS) three times and stored in $70 \%$ ethanol prior to scanning. The fixed tibiae were then analyzed using a high-resolution micro-CT (Skyscan 1072; Skyscan, Aartselaar, Belgium). The scanning protocol was set at an isometric resolution of $9 \mu \mathrm{m}$, and X-ray energy settings of $70 \mathrm{kV}$ and $80 \mu \mathrm{A}$. After reconstruction, the region of interest of the tibia was set at $0.5 \mathrm{~mm}$ from the proximal growth plate and selected for further qualitative and quantitative analysis. Several structural parameters, including trabecular bone volume per total volume (BV/TV), mean trabecular thickness (Tb.Th), mean trabecular number (Tb.N) and mean trabecular separation (Tb.Sp) were measured by micro-CT as reported previously (16).

Microarray analysis. Total RNA was isolated from BMMs that had been treated with RANKL for 0 or 5 days using the RNeasy ${ }^{\circledR}$ Mini Kit (Invitrogen; Thermo Fisher Scientific, Inc.). The samples were then submitted to LC Sciences (Houston, TX, USA) for microarray analysis. The probe content was consistent with the miRBase database version 14.0 (http://www.mirbase.org/). The fluorescence value was determined by subtracting the background. The ratio of the two subgroups ( $\log 2$ transformed) and P-values were calculated using Student's t-test. MiRNAs with $\mathrm{P}<0.01$ and fluorescence values $>500$ were selected.

Reverse-transcription quantitative polymerase chain reaction (RT-qPCR) analysis. Homogenized tissue was obtained by grinding with liquid nitrogen. Total RNA (including miRNA) was isolated from cells and tissues by using the RNeasy ${ }^{\circledR}$ Mini kit (Invitrogen; Thermo Fisher Scientific, Inc.) and reverse transcription of $1.0 \mu \mathrm{g}$ total RNA was performed using the miRNA cDNA kit or the HiFiScript cDNA kit (CWBIO, Beijing, China), which were used to analyze the expression levels of miRNA and osteoclast-specific genes, respectively. Amplification reactions were set up in 20- $\mu$ l reaction volumes containing amplification primers and UltraSYBR Mixture (with ROX) (CWBIO), and products were detected using an ABI 7500 sequencing detection system (Thermo Fisher Scientific, Inc.). A 1- $\mu l$ volume of cDNA and $1-\mu 1$ of primers (Sangon Biotech Co., Ltd., Shanghai, China) were used in each amplification reaction. The conditions of the reaction were as follows: Activation at $95^{\circ} \mathrm{C}$ for $10 \mathrm{~min}$; 40 cycles of amplification, $95^{\circ} \mathrm{C}$ for $10 \mathrm{sec}, 60^{\circ} \mathrm{C}$ for $24 \mathrm{sec}$ and $72^{\circ} \mathrm{C}$ for $20 \mathrm{sec}$; and final extension at $72^{\circ} \mathrm{C}$ for $1 \mathrm{~min}$. All reactions were run in triplicate and were normalized to the miRNA house-keeping gene U6, or mRNA house-keeping gene $18 \mathrm{~S}$ and glyceraldehyde-3-phosphate dehydrogenase (GAPDH). Expression was quantified using the $\Delta \mathrm{Cq}$ method, as described previously (17).

Primer sequences were as follows: $18 \mathrm{~S}$ forward, 5 '-CCT GCGGCTTAATTTGACTC-3' and reverse, 5'-AACTAA GAACGGCCATGCAC-3'; GAPDH forward, 5'-ACCCAG AAGACTGTGGATGG-3' and reverse, 5'-CACATTGGG GGTAGGAACAC-3'; TRAP forward, 5'-CCATTGTTAGCC ACATACGG-3' and reverse, 5'-CACTCAGCACATAGC CCACA-3'; Ctsk forward, 5'-TCCGCAATCCTTACCGAA TA-3' and reverse, 5'-AACTTGAACACCCACATCCTG-3'; NFATc1 forward, 5'-TCCACCCACTTCTGACTTCC-3' and reverse, 5'-CTTCGCCCACTGATACGAG-3'; U6 forward, 5'-CTCGCTTCGGCAGCACA-3' and reverse, 5'-AACGCT TCACGAATTTGCGT-3'; mmu-miR-27 forward, 5'-GTG GTTCACAGTGGCTAAG-3'; mmu-miR-21a-5p forward, 5'-GTTTGGTAGCTTATCAGACTGA-3'; mmu-let-7i-5p forward, 5'-GTT TGG TGAGGT AGT AGT TTGT-3'; mmu-miR-22-3p forward, 5'-GTGAAGCTGCCAGTT GAAG-3'; mmu-miR-378-3p forward, 5'-GTTGACTGGACT 
Table I. MiRs with fold-changes of $>1.5$ during osteoclastogenesis between days 0 and 5 according to microarray analysis.

\begin{tabular}{lcc}
\hline Probe ID & Fold change (day 5 vs. 0) & Standard deviation \\
\hline mmu-miR-22-3p & 1.53 & 0.13 \\
mmu-miR-21-5p & 2.02 & 0.23 \\
mmu-miR-27a-3p & 1.57 & 0.35 \\
mmu-let-7i-5p & 1.64 & 0.33 \\
mmu-miR-340-5p & 1.70 & 0.18 \\
mmu-miR-23a-5p & 3.87 & 0.08
\end{tabular}

miR, microRNA.

A
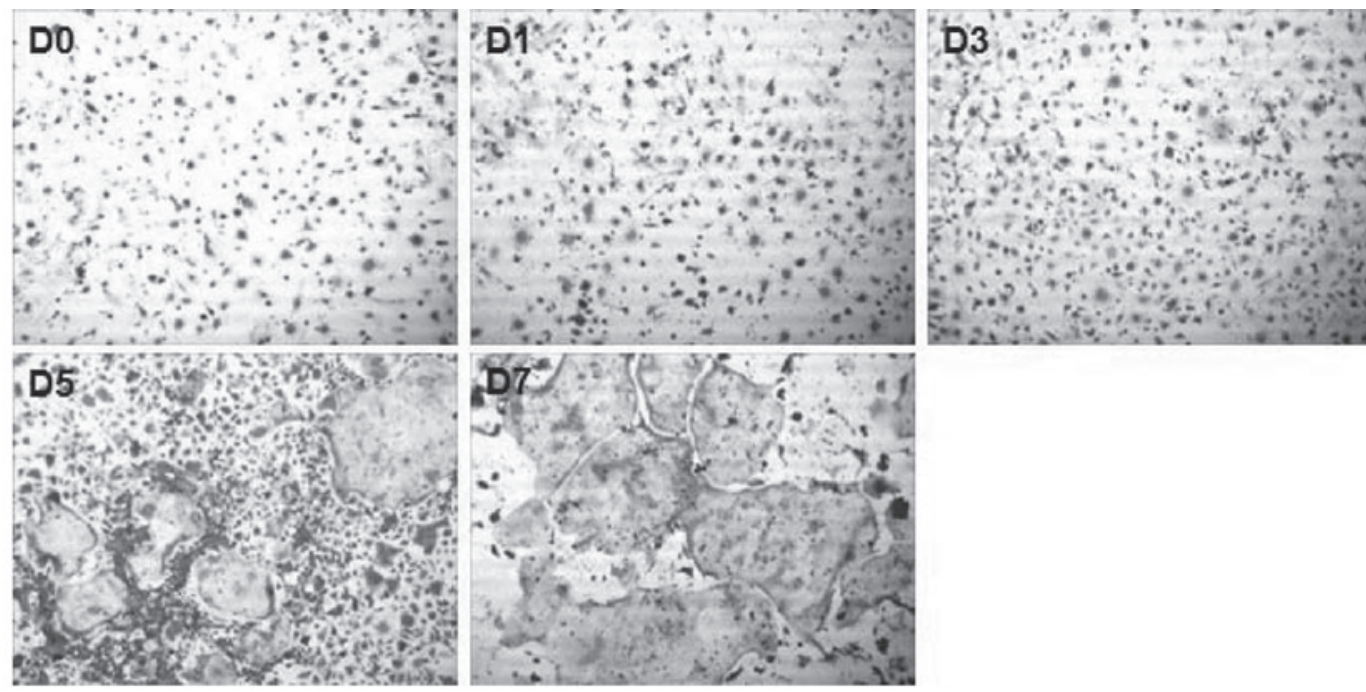

B

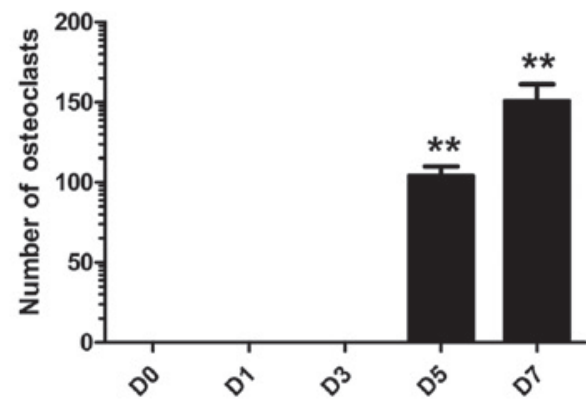

C
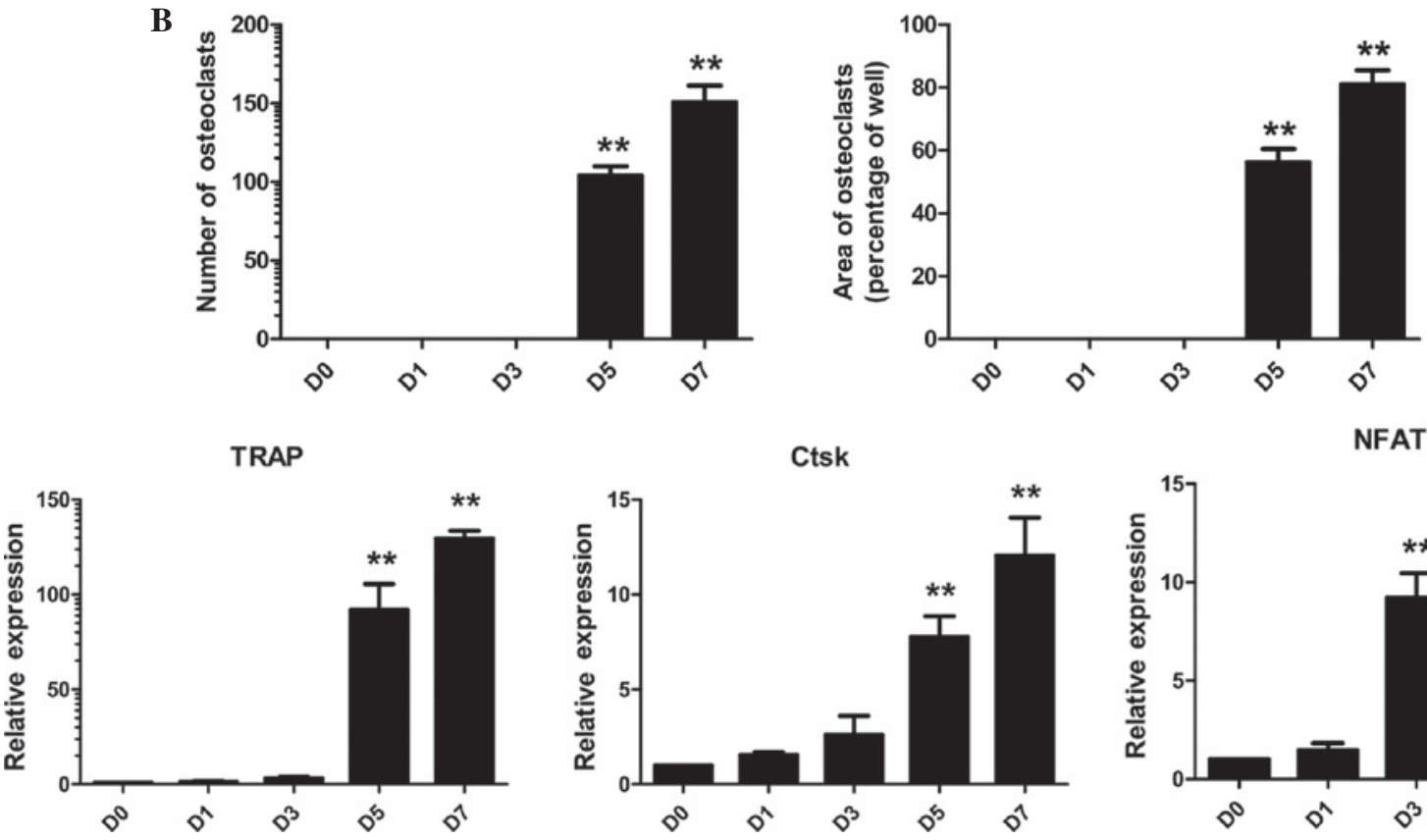

Ctsk

NFATc1

Figure 1. Validation of osteoclastogenesis in vitro. (A) Bone marrow macrophages were treated with $30 \mathrm{ng} / \mathrm{ml} \mathrm{macrophage} \mathrm{colony-stimulating} \mathrm{factor} \mathrm{in} \mathrm{the}$ presence of $50 \mathrm{ng} / \mathrm{ml}$ receptor activator of nuclear factor- $\mathrm{B}$ ligand for $0,1,3,5$ or 7 days. Cells were then fixed with $4 \%$ paraformaldehyde and subjected to TRAP staining (magnification, x40). (B) The number and area of TRAP-positive multinuclear cells were determined. (C) The expression of osteoclast-specific genes, including Ctsk, NFATc1 and TRAP, were detected by quantitative polymerase chain reaction. Values are expressed as mean \pm standard error of the mean. ${ }^{* * *} \mathrm{P}<0.01$ vs. D0. TRAP, tartrate-resistant acid phosphatase; D, day.

TGGAGTC-3'; mmu-miR-340-5p forward, 5'-GTGGGGTTA TAAAGCAATGAGA-3'; mmu-miR-23a-5p forward, 5'-TTG
GGGGTTCCTGGGGAT-3'; common reverse, GTGCAG GGTCCGAGGT. 
Table II. miRs with a known function in osteoclasts and aberrant expression during ostoclastogenesis.

Expression

\begin{tabular}{lllclc} 
miR & Function & Present study & Fold change (day 5 vs. 0) & Previous study & Reference \\
\hline miR-223 & Suppression of NFI-A & Downregulated & 0.191 & Downregulated & 18 \\
miR-155 & Suppression of MITF and SOCS1 & Downregulated & 0.017 & Downregulated & 19 \\
miR-296 & Suppression of c-Fos and MMP2 & Downregulated & 0.756 & Downregulated & 11 \\
miR-21 & Suppression of PDCD4 & Downregulated & 0.494 & Upregulated & 12 \\
miR-146a & Suppression of TRAF6 & Downregulated & 0.351 & Downregulated & 20 \\
miR-133a & Suppression of MITF and MMP14 & No change & 1.000 & Downregulated & 21 \\
miR-219 & Suppression of MITF and TRAF6 & No change & 1.000 & Downregulated & 21 \\
miR-148a & Suppression of MAFB & Upregulated & 2.178 & Upregulated & 22 \\
miR-124 & Suppression of NFATc1 & No change & 1.000 & Downregulated & 23 \\
miR-31 & Suppression of RhoA & No change & 1.000 & Upregulated & 24 \\
miR-503 & Suppression of RANK & Downregulated & 0.030 & Downregulated & 25 \\
miR-125a & Suppression of TRAF6 & Downregulated & 0.772 & Downregulated & 26 \\
miR-141 & Suppression of MITF and CALCR & No change & 1.000 & Downregulated & 21 \\
miR-190 & Suppression of CALCR & No change & 1.000 & Downregulated & 21 \\
miR-34a & Suppression of Tgif2 & Downregulated & 0.801 & Downregulated & 27
\end{tabular}

miR, microRNA.
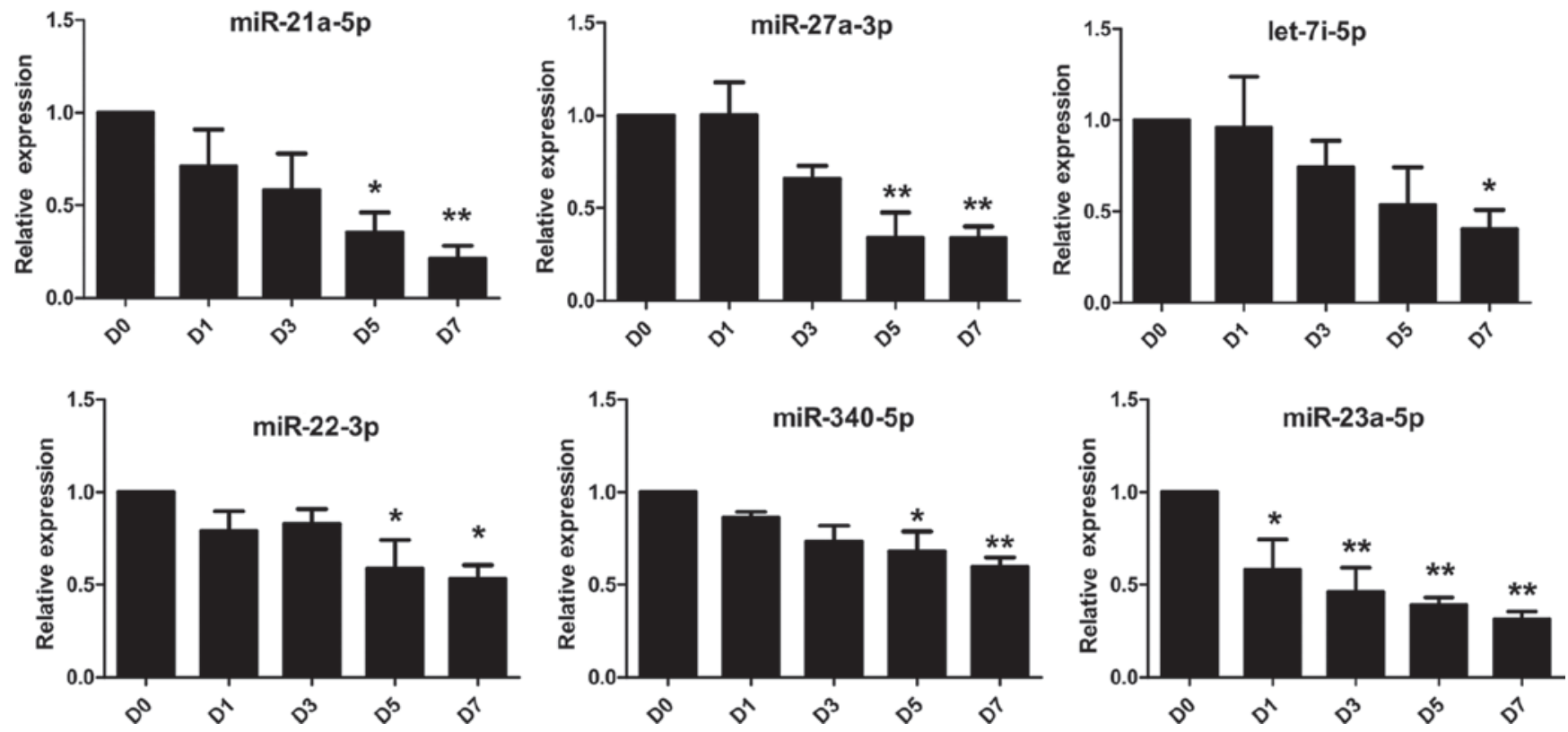

Figure 2. Six miRNAs were downregulated during osteoclast differentiation. Expression profiles of miRNAs during osteoclastogenesis were detected by reverse-transcription quantitative polymerase chain reaction analysis. Values are expressed as mean \pm standard error of the mean. ${ }^{*} \mathrm{P}<0.05$, ${ }^{* * *} \mathrm{P}<0.01 \mathrm{vs}$. D0. miR, microRNA; D, day.

Statistical analysis. GraphPad Prism ${ }^{\circledast}$ software (GraphPad Inc., La Jolla, CA, USA) was used for all the statistical analyses. Values are expressed as the mean \pm standard error of the mean. Analysis of variance followed by Dunnett's post hoc test was used for comparisons between groups. All the experiments were performed at least three times and $\mathrm{P}<0.05$ was considered to indicate a statistically significant difference.

\section{Results}

Validation of osteoclastogenesis in vitro. In order to obtain miRNAs during various stages of osteoclast differentiation, BMMs were treated with M-CSF and RANKL for $0,1,3,5$ or 7 days. As presented in Fig. 1A, BMMs were induced into mature osteoclasts following treatment with RANKL for 5 or 7 days. While no osteoclasts were present 
A

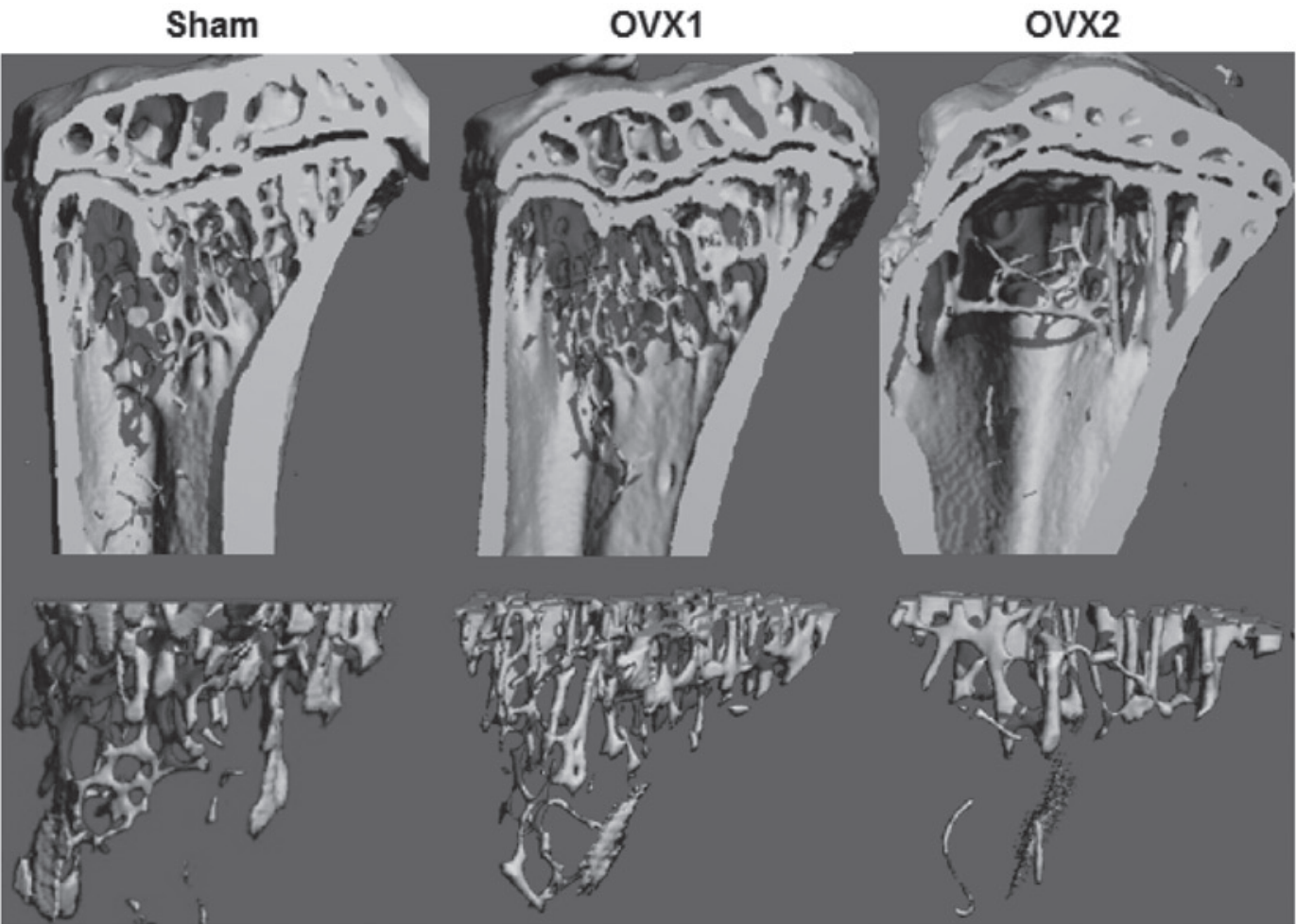

$\mathbf{B}$
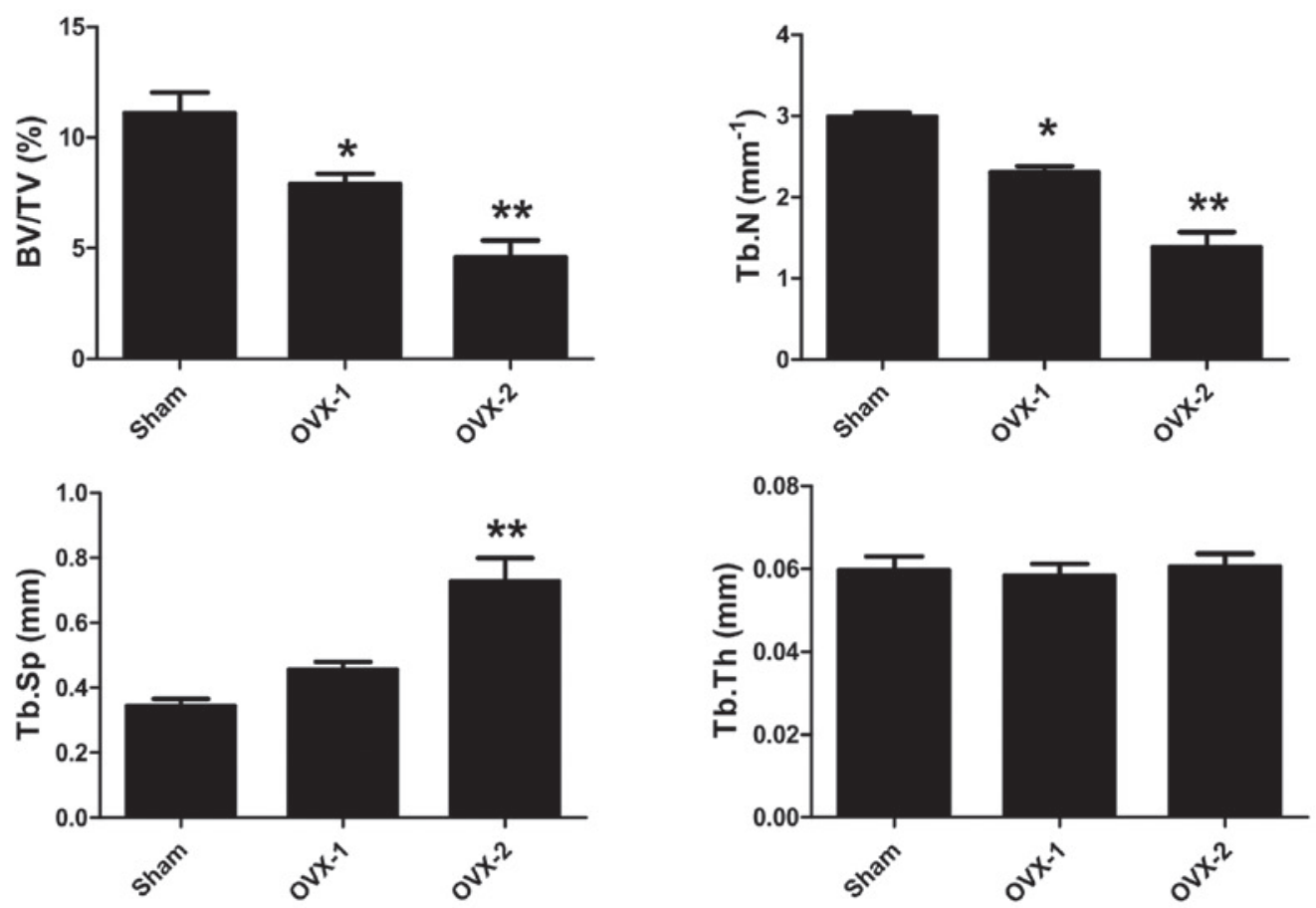

Figure 3. Generation of an OVX-induced osteoporosis model with various degrees of bone loss. (A) The fixed tibiae were analyzed by micro-computed tomography and three-dimensionally reconstructed images from each group are shown (magnification, $\mathrm{x} 40$ ). (B) For each sample, BV/TV, Tb.Th, Tb.N and Tb.Sp were measured. Values are expressed as mean \pm standard error of the mean. ${ }^{*} \mathrm{P}<0.05,{ }^{* *} \mathrm{P}<0.01$ vs. the sham group. BV, trabecular bone volume; TV, total volume; Tb.Th, mean trabecular thickness; Tb.N, mean trabecular number; Tb.Sp, mean trabecular separation; OVX1, mice at 1 month following OVX; OVX2, mice at 2 months following OVX; OVX, ovariectomy.

at days 0-3, the number of osteoclasts (TRAP-positive cells with $>3$ nuclei) was $103.93 \pm 3.55$ per well and $150.67 \pm 6.13$ per well following treatment of RANKL for 5 and 7 days, respectively (Fig. 1B). Furthermore, the area of osteoclasts was $56.30 \pm 2.43 \mu \mathrm{m}^{2}$ and $81.03 \pm 2.56 \mu \mathrm{m}^{2}$, respectively, following treatment with RANKL for 5 and 7 days (Fig. 1B).
In addition, the expression levels of osteoclast-specific genes Ctsk, NFATc1 and TRAP were also assessed. As expected, the osteoclast-associated genes were upregulated in response to stimulation with RANKL. NFATc1 levels peaked at day three, while the expression of TRAP and Ctsk was low until day 3 and significantly increased on day 5 with a further 

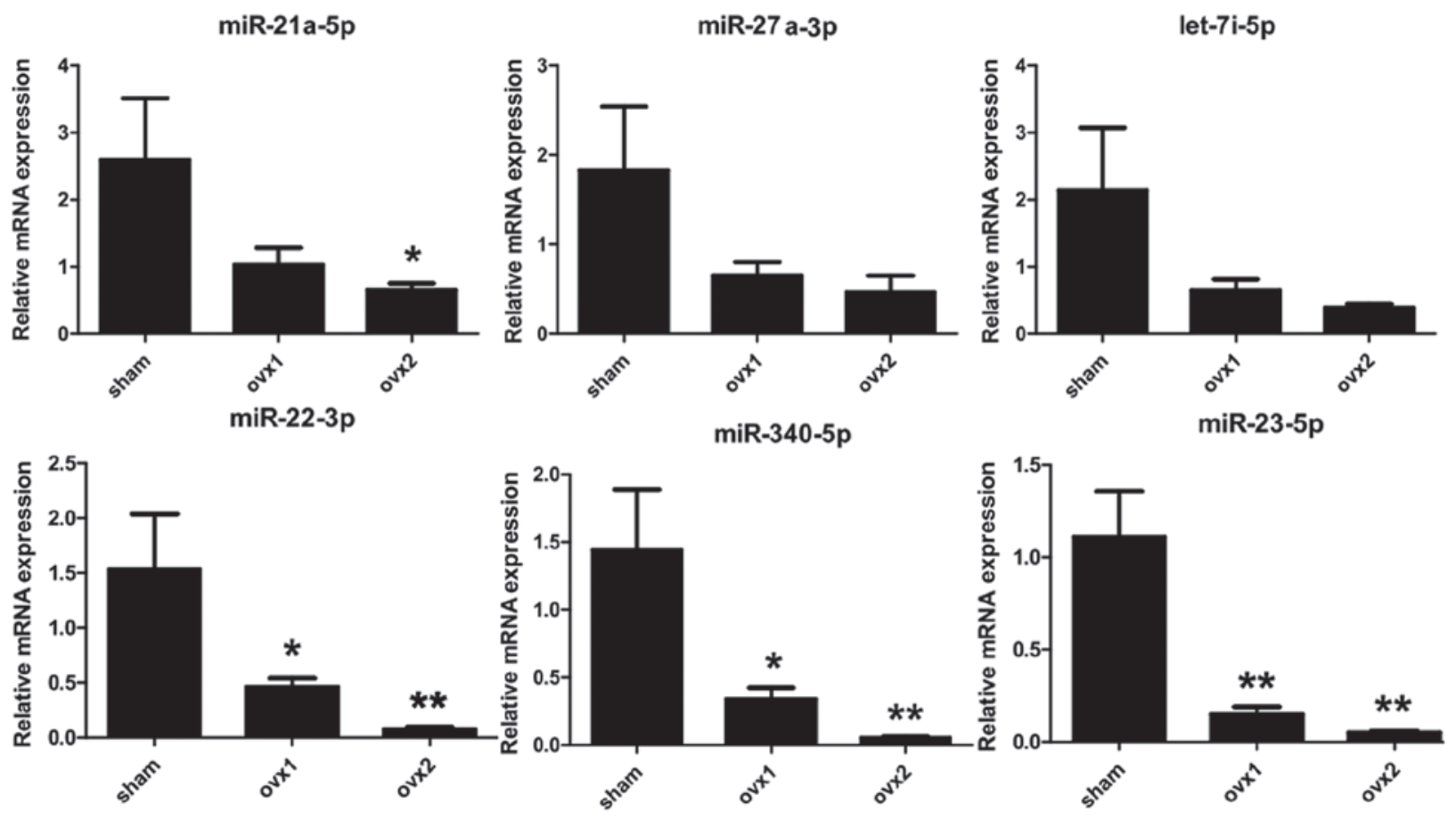

Figure 4. Differently expressed miRNAs in OVX mice as determined by reverse-transcription quantitative polymerase chain reaction analysis. Values are expressed as the mean \pm standard error of the mean. ${ }^{*} \mathrm{P}<0.05,{ }^{* *} \mathrm{P}<0.01$ vs. the sham group. miR, microRNA; OVX, ovariectomy; OVX1, mice at 1 month following OVX; OVX2, mice at 2 months following OVX.

increase on day 7 (Fig. 1C). These results suggested that osteoclast maturation follows stimulation with RANKL for 5-7 days.

Downregulated miRNAs during osteoclast differentiation. Based on the successful induction of osteoclasts, the expression of miRNAs during osteoclastogenesis was investigated using total RNA extracted from BMMs (day 0) and mature osteoclasts (day 5) using microarray analysis. As listed in Table I, six miRNAs that were downregulated during osteoclastogenesis with a fold change of $>1.5$ were identified, including miR-340-5p, miR-23a-5p, miR-22-3p, miR-21-5p, miR-27a-3p and let-7i-5p. Largely consistent with the results of previous studies, the downregulation of further miRNAs, including miR-21, miR-155, miR-223 and miR-146a, was identified by microarray analysis (presented in Table II) $(11,12,18-27)$.

Furthermore, changes in the expression of the six aberrantly expressed miRNAs during osteoclastogenesis was confirmed using RT-qPCR. As presented in Fig. 2, miR-21a-5p, miR-27a-3p, let-7i-5p, miR-22-3p, miR-340-5p and miR-23a-5p were observed to be downregulated during osteoclast differentiation. The expression of miR-27a-3p, miR-21a-5p and miR-23a-5p in matured osteoclasts was demonstrated to be $\sim 70-80 \%$ lower than that in BMMs without RANKL stimulation, while the expression of let-7i-5p and miR-22-3p dropped to 50 70\% in BMMs following treatment with RANKL for 5 or 7 days. Notably, the expression profiles of these miRNAs demonstrated a time-dependent decrease during osteoclast formation. These results confirmed the downregulation of a number of miRNAs during osteoclastogenesis.
Generation of an OVX-induced osteoporosis model with various degree of bone loss. An OVX-induced murine osteoporosis model was used to establish various degrees of osteoporosis in vivo. Microarchitectural changes of the tibiae were then detected by micro-CT (Fig. 3A). In accordance with other studies (21), OVX mice showed lower BV/TV and Tb.N, and higher Tb.Sp compared to sham-operated mice (Fig. 3B). In addition, the mice sacrificed two months after ovariectomy (OVX 2 group) displayed a reduced bone volume compared with that of the mice in the OVX 1 group, with BV/TVs of $7.89 \pm 0.27$ and $4.60 \pm 0.44 \%$, respectively (Fig. 3B). In addition, OVX 2 mice exhibited a lower Tb.N and a higher Tb.Sp than the OVX 1 mice (Fig. 3B). The Tb.Th value remained constant indicating that OVX-induced osteoporosis occurred predominantly by decreasing BV/TV and Tb.N. The changes in the factors of bone intactness indicated that the degree of osteoporosis in OVX 2 mice was higher than that in OVX 1 mice.

Osteoclastogenesis-associated miRNAs are aberrantly expressed in OVX mice. Based on the expression of miRNAs during osteoclast differentiation according to the microarray and RT-qPCR analyses of the present study, the expression of these miRNAs was assessed in the tibiae of a mouse model of osteoporosis. As shown in Fig. 4, miR-21a-5p, miR-27a-3p, let-7i-5p, miR-22-3p, miR-340-5p and miR-23a-5p were found to be downregulated in OVX 1 mice by at least $60 \%$ when compared to the sham-operated mice. Of note, the expression of all of these miRNAs was further declined in OVX 2 mice when compared to that in OVX 1 mice, indicating a time-dependent decrease in the expression of these miRNAs during the progression of osteoporosis. 


\section{Discussion}

The present study used a microarray assay to identify that miR-21a-5p, miR-27a-3p, let-7i-5p, miR-22-3p, miR-340-5p and miR-23a-5p were downregulated during osteoclastogenesis. This result was further supported by RT-qPCR analysis of BMMs induced to undergo osteoclastogenesis by stimulation with RANKL in vitro as well as in a murine model of progressive osteoporosis in vivo. Of note, the expression of these miRNAs was associated with the progression of osteoporosis, with lower expression in more severe osteoporosis. These miRNAs may represent potential biomarkers and targets for the diagnosis and treatment of osteolytic diseases such as osteoporosis.

However, the present study had several limitations. First, osteoclasts are not the only type of cell in bone tissue. The expression of miRNAs in the tibiae of the in vivo model represents the expression levels in whole bone tissue, including osteoclasts, osteoblasts and osteocytes. A more significant decrease of these miRNAs was observed in vivo compared with that obtained in vitro. Thus, it can be assumed that the expression of these miRNAs may also have been altered in osteoblasts and osteocytes during the development and progression of osteoporosis.

The functional role of the miRNAs identified in the present study in osteoclasts requires further elucidation. In previous studies, the expression of miR-27a-3p was increased during odontoblastic differentiation of hFOB1.19 as well as MDPC-23 cells $(28,29)$. By regulating the APC gene, miR-27 can affect $\beta$-catenin accumulation during osteoblast and odontoblast differentiation (28). However, whether the downregulation of miR-27 in osteoclasts is involved in the wnt/ $\beta$-catenin signaling pathway requires further research. Similarly, miR-22 was associated with rheumatoid arthritis via a novel p53/miR-22/cysteine-rich angiogenic inducer 61 axis in synovial cells (30). In hepatocytes, miR-22 significantly suppressed nuclear factor- $\kappa \mathrm{B}(\mathrm{NF}-\kappa \mathrm{B})$ activity by regulating the expression of nuclear receptor co-activator 1 and nuclear receptor-interacting protein 1, two NF- $\kappa \mathrm{B}$ activators (31). However, the regulation of NF- $\mathrm{NB}$ signaling by miR-22-3p during osteoclastogenesis requires further investigation. In addition, miR-340 was reported to regulate $\mathrm{RAS} / \mathrm{RAF} /$ mitogen-activated protein kinase (MAPK) signaling by modulating the expression of multiple components of this pathway in melanoma (32). As the activation of the MAPK pathway is crucial in RANKL-induced osteoclastogenesis, miR-340-5p is likely to have a particular function in osteoclasts. In general, further functional analysis of these miRNAs in osteoclasts is required to reveal the underlying molecular mechanisms.

In conclusion, the present study identified six aberrantly expressed miRNAs in BMMs undergoing osteoclastogenesis and in mice with OVX-induced osteoporosis. The expression of these miRNAs was shown to be associated with the degree of bone loss in osteoporosis. The identified miRNAs miR-21a-5p, miR-27a-3p, let-7i-5p, miR-22-3p, miR-340-5p and miR-23a-5p are therefore of potential diagnostic and therapeutic value for osteolytic diseases.

\section{Acknowledgements}

The present study was supported by the National Nature Science Fund of China (nos. 81271971, 81271972 and 31270997).

\section{References}

1. Rachner TD, Khosla S and Hofbauer LC: Osteoporosis: Now and the future. Lancet 377: 1276-1287, 2011

2. Kim MS, Magno CL, Day CJ and Morrison NA: Induction of chemokines and chemokine receptors CCR2b and CCR4 in authentic human osteoclasts differentiated with RANKL and osteoclast like cells differentiated by MCP-1 and RANTES. J Cell Biochem 97: 512-518, 2006.

3. Matsuo K and Irie N: Osteoclast-osteoblast communication. Arch Biochem Biophys 473: 201-209, 2008.

4. Dempster DW, Lambing CL, Kostenuik PJ and Grauer A: Role of RANK ligand and denosumab, a targeted RANK ligand inhibitor, in bone health and osteoporosis: A review of preclinical and clinical data. Clin Ther 34: 521-536, 2012.

5. Chong Y, Zhang J, Guo X, Li G, Zhang S, Li C, Jiao Z and Shao M: MicroRNA-503 acts as a tumor suppressor in osteosarcoma by targeting L1CAM. PLoS One 9: e114585, 2014.

6. Kloos W, Vogel B and Blessing E: MiRNAs in peripheral artery disease-something gripping this way comes. Vasa 43: 163-170, 2014.

7. Schneider MR: MicroRNAs as novel players in skin development, homeostasis and disease. Br J Dermatol 166: 22-28, 2012.

8. Jayaswal V,Lutherborrow M, Ma DD and Yang YH: Identification of microRNA-mRNA modules using microarray data. BMC Genomics 12: 138, 2011.

9. Sugatani T and Hruska KA: MicroRNA-223 is a key factor in osteoclast differentiation. J Cell Biochem 101: 996-999, 2007.

10. Bluml S, Bonelli M, Niederreiter B, Puchner A, Mayr G, Hayer S, Koenders MI, van den Berg WB, Smolen J and Redlich K: Essential role of microRNA-155 in the pathogenesis of autoimmune arthritis in mice. Arthritis Rheum 63: 1281-1288, 2011.

11. Rossi M, Pitari MR, Amodio N, Di Martino MT, Conforti F, Leone E, Botta C, Paolino FM, Del Giudice T, Iuliano E, et al: miR-29b negatively regulates human osteoclastic cell differentiation and function: Implications for the treatment of multiple myeloma-related bone disease. J Cell Physiol 228: 1506-1515, 2013.

12. Sugatani T, Vacher J and Hruska KA: A microRNA expression signature of osteoclastogenesis. Blood 117: 3648-3657, 2011.

13. Krzeszinski JY, Wei W, Huynh H, Jin Z, Wang X, Chang TC, Xie XJ, He L, Mangala LS, Lopez-Berestein G, et al: miR-34a blocks osteoporosis and bone metastasis by inhibiting osteoclastogenesis and Tgif2. Nature 512: 431-435, 2014.

14. Seeliger C, Karpinski K, Haug AT, Vester H, Schmitt A, Bauer JS and van Griensven M: Five freely circulating miRNAs and bone tissue miRNAs are associated with osteoporotic fractures. J Bone Miner Res 29: 1718-1728, 2014.

15. Li H, Zhai Z, Liu G, Tang T, Lin Z, Zheng M, Qin A and Dai K: Sanguinarine inhibits osteoclast formation and bone resorption via suppressing RANKL-induced activation of NF-kB and ERK signaling pathways. Biochem Biophys Res Commun 430: 951-956, 2013.

16. Bouxsein ML, Boyd SK, Christiansen BA, Guldberg RE, Jepsen KJ and Müller R: Guidelines for assessment of bone microstructure in rodents using micro-computed tomography. J Bone Miner Res 25: 1468-1486, 2010.

17. Imai T, et al: Evaluation of reference genes for accurate normalization of gene expression for real time-quantitative PCR in Pyrus pyrifolia using different tissue samples and seasonal conditions. PLoS One 9: e86492, 2014.

18. Shibuya H, Nakasa T, Adachi N, Nagata Y, Ishikawa M, Deie M, Suzuki O and Ochi M: Overexpression of microRNA-223 in rheumatoid arthritis synovium controls osteoclast differentiation. Mod Rheumatol 23: 674-685, 2013.

19. Zhang J, Zhao H, Chen J, Xia B, Jin Y, Wei W, Shen J and Huang Y: Interferon- $\beta$-induced miR-155 inhibits osteoclast differentiation by targeting SOCS1 and MITF. FEBS Lett 586: 3255-3262, 2012.

20. Nakasa T, Shibuya H, Nagata Y, Niimoto T and Ochi M: The inhibitory effect of microRNA-146a expression on bone destruction in collagen-induced arthritis. Arthritis Rheum 63: 1582-1590, 2011.

21. Ell B, Mercatali L, Ibrahim T, Campbell N, Schwarzenbach H, Pantel K, Amadori D and Kang Y: Tumor-induced osteoclast miRNA changes as regulators and biomarkers of osteolytic bone metastasis. Cancer Cell 24: 542-556, 2013.

22. Cheng P, Chen C, He HB, Hu R, Zhou HD, Xie H, Zhu W, Dai RC, Wu XP, Liao EY and Luo XH: miR-148a regulates osteoclastogenesis by targeting V-maf musculoaponeurotic fibrosarcoma oncogene homolog B. J Bone Miner Res 28: 1180-1190, 2013. 
23. Lee Y, Kim HJ, Park CK, Kim YG, Lee HJ, Kim JY and Kim HH: MicroRNA-124 regulates osteoclast differentiation. Bone 56 : 383-389, 2013.

24. Mizoguchi F, Murakami Y, Saito T, Miyasaka N and Kohsaka H: miR-31 controls osteoclast formation and bone resorption by targeting RhoA. Arthritis Res Ther 15: R102, 2013.

25. Chen C, Cheng P, Xie H, Zhou HD, Wu XP, Liao EY and Luo XH: MiR-503 regulates osteoclastogenesis via targeting RANK. J Bone Miner Res 29: 338-347, 2014.

26. Guo LJ, Liao L, Yang L, Li Y and Jiang TJ: MiR-125a TNF receptor-associated factor 6 to inhibit osteoclastogenesis. Exp Cell Res 321: 142-152, 2014.

27. Wu S, Huang S, Ding J, Zhao Y, Liang L, Liu T, Zhan R and He X: Multiple microRNAs modulate $\mathrm{p} 21 \mathrm{Cip} 1 /$ Waf1 expression by directly targeting its 3'untranslated region. Oncogene 29: 2302-2308, 2010.

28. Park MG, Kim JS, Park SY, Lee SA, Kim HJ, Kim CS, Kim JS, Chun HS, Park JC and Kim do K: MicroRNA-27 promotes the differentiation of odontoblastic cell by targeting APC and activating Wnt/ $\beta$-catenin signaling. Gene 538: 266-272, 2014.
29. Wang T and Xu Z: miR-27 promotes osteoblast differentiation by modulating Wnt signaling. Biochem Biophys Res Commun 402: 186-189, 2010.

30. Lin J, Huo R, Xiao L, Zhu X, Xie J, Sun S, He Y, Zhang J, Sun Y, Zhou Z, et al: A novel p53/microRNA-22/Cyr61 axis in synovial cells regulates inflammation in rheumatoid arthritis. Arthritis Rheumatol 66: 49-59, 2014.

31. Takata A, Otsuka M, Kojima K, Yoshikawa T, Kishikawa T, Yoshida $\mathrm{H}$ and Koike K: MicroRNA-22 and microRNA-140 suppress NF-kB activity by regulating the expression of NF-kB coactivators. Biochem Biophys Res Commun 411: 826-831, 2011.

32. Poenitzsch Strong AM, Setaluri V and Spiegelman VS: microRNA-340 as a modulator of RAS-RAF-MAPK signaling in melanoma. Arch Biochem Biophys 563: 118-124, 2014. 